\section{Redes atm de alto desempenho}

Flávia Oliveira Santos de Sá Lisboa*

\section{Introdução}

O desenvolvimento da tecnologia ATM (Asynchronous Transfer Mode) tinha como principais objetivos integrar em uma única rede serviços de voz, vídeo e dados de maneira eficiente, usando os mesmos meios físicos; aumentar largura de banda, principalmente devido ao crescimento da Internet que passou a requerer mais largura de banda para a transmissão de dados tão complexos aumentar velocidade nas transmissões, principalmente, fazendo uso eficiente de fibras óticas. Por ter sido desenvolvida a fim de atingir tais objetivos, a tecnologia ATM vem sendo utilizada na implementação de backbone de LANs e WANs, justamente por oferecer a possibilidade de integração de serviços com qualidade, alta escalabilidade e altas taxas de transferência em banda larga.

Este artigo abrange um breve histórico sobre a tecnologia de redes ATM enfatizando as idéias que motivaram seu desenvolvimento. Apresenta os conceitos básicos da tecnologia, com as principais características que a definem, fazendo comparações com a tecnologia Ethernet (tecnologia de rede mais conhecida atualmente para redes locais) a fim de delinear melhor tais características. Alguns casos de empresas e instituições que utilizam ATM em suas redes são abordados e como considerações finais um quadro ilustra um comparativo entre o ATM e a tecnologia Gigabit Ethernet, ressaltando os pontos fortes de cada uma.

\section{Breve histórico da tecnologia ATM}

A tecnologia ATM surgiu da necessidade crescente da integração de serviços de voz, vídeo e grandes volumes de dados, aliado à implantação em larga escala de fibras ópticas. Esta tecnologia é capaz de fornecer um formato comum de comunicação para serviços com tão diferentes necessidades de largura de banda. Ela é caracterizada por uma filosofia revolucionária de comutação de células de

* Professora de Informática nos cursos de Administração e Economia na UNIARA. Analista de Sistemas no Instituto de Física de São Carlos da Universidade de São Paulo. Mestre em Ciência da Computação pela Universidade Federal de São Carlos tamanho fixo através de canais virtuais estabelecidos sob demanda, diferentemente das redes tradicionais que se preocupam em fazer o melhor possível para que os datagramas cheguem ao seu destino através do roteamento de datagramas.

Tudo começou quando grupos de padronização (como o ITU-T, atualmente CCITT) iniciaram pesquisas nos anos 80 (Web ProForums, 2004) com o objetivo de estabelecer critérios para transmissão, chaveamento, sinalização e técnicas de controle necessárias para se implementar uma tecnologia de rede inteligente baseada em fibra óptica. Tal tecnologia deveria solucionar limitações encontradas até então e permitir que a rede pudesse ser capaz de transmissões eficientes de serviços de voz, vídeo e dados. Em princípio, esta rede foi nomeada Broadband Integrated Services Digital Network (BISDN) ou Redes Digitais de Serviços Integrados de Faixa Larga (RDSI-FL). Em 1990, as RDSI-FL passaram a ser baseadas no método de transferência SONET/SDH (Synchronous Optical Network/Synchronous Digital Hierarchy). Como tal método utilizava o modo síncrono de transferência, essa tecnologia passou a ser conhecida como STM (Synchronous Transfer Mode).

O modo síncrono de transferência caracteriza-se pela comutação de circuitos com alocação de banda fixa para todos os circuitos abertos. Como a demanda dos circuitos pode ser diferente, algumas bandas alocadas podem ficar subtilizadas pois existem aplicações que não necessitam de toda a largura de banda alocada. Por este motivo, foi, então, adotado o modo assíncrono de transferência que se caracteriza pela comutação de pequenos pacotes (células) com alocação de largura de banda conforme a demanda. Isto quer dizer que só será alocada a banda se a aplicação realmente necessitar. Em 1992, é especificada a tecnologia ATM que se baseava no modo de transferência assíncrono. Podemos entender o ATM como uma evolução do STM por utilizar um método de transferência mais eficiente.

$\mathrm{O}$ quadro abaixo apresenta os principais marcos que possibilitaram o desenvolvimento da tecnologia ATM (ATM Forum, 2004).

\begin{tabular}{|c|c|}
\hline 1980 & Inicio das pesquisas sobre comunicaço de redes de fibra óptica usando pacotes. \\
\hline 986 & Grupo de padronizasāo ITU-T (atualmethe CCITT) adota a fede denominada RDSI-FL. \\
\hline 1989 & Adoçâo do esquema de ólualas de 53 bytes. \\
\hline 1991 & 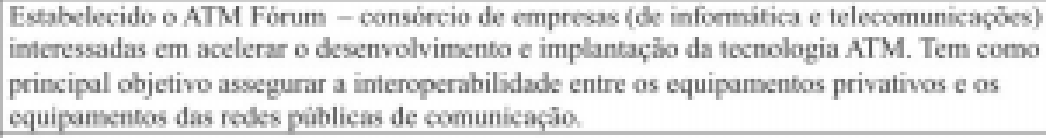 \\
\hline & Primeira ediçầo da tecnologia ATM é especificada \\
\hline
\end{tabular}

\section{Visão geral do ATM}

A tecnologia ATM se fundamenta em três características essenciais que formam toda a sua base: parâmetros de Qualidade de Serviço (QoS), células de tamanho pequeno e fixo e tecnologia orientada à conexão. 
A primeira característica é que ATM é uma tecnologia desenvolvida para ser uma rede única integrando serviços de dados, voz e vídeo; mas o mais importante é a integração com qualidade e garantia nas transmissões. Para isto, o ATM compreende parâmetros de qualidade de serviço através de recursos de QoS (Quality of Service) que o ATM tem nativo. Atualmente, o padrão Gigabit Ethernet também está desenvolvendo classes de serviço de qualidade, mas no Ethernet o QoS não é nativo e, portanto, não é tão eficiente quanto o QoS implementado pelo ATM.

A segunda característica é que o ATM se baseia na transmissão de células de 53 bytes de tamanho fixo, em que 5 bytes são de cabeçalho e os 48 bytes restantes são os dados. No ATM a unidade de informação (UI) é a célula ATM, no Ethernet a UI é o pacote Ethernet. As células ATM têm sempre o mesmo tamanho de 53 bytes. Já os pacotes Ethernet são variáveis e de tamanho grande. O pacote Ethernet típico tem 26 bytes de cabeçalho e pode ter de 46 a 1500 bytes de dados. As vantagens de se ter células de tamanho pequeno e fixo são abordadas na próxima seção deste artigo.

Uma terceira característica é que o ATM é, fundamentalmente, orientado a conexão. Isto quer dizer que uma conexão virtual deve ser estabelecida antes de se iniciar a transmissão dos dados. O ATM funciona com a criação de circuitos virtuais que permitem que se tenha um caminho definido da origem para o destino por onde as células devem ser transmitidas. Com o circuito virtual criado, as células podem seguir sempre pelo mesmo caminho de maneira ordenada. Já o padrão Ethernet segue a linha do "melhor esforço", em que a rede faz o possível para entregar o pacote no seu destino. Desta forma os pacotes não necessariamente seguem pelo mesmo caminho, podendo chega fora de ordem, o que torna necessário o reordenamento dos pacotes no destino, causando mais atrasos nas transmissões.

Essas três características tornam o ATM uma boa alternativa para ser utilizado em redes locais, redes metropolitana e de longa distância sem distinções, principalmente porque:

- conferem alta escalabilidade, no que se refere ao aumento do número de nós na rede e também aumento de largura de banda;

- conferem baixa latência/atrasos, principalmente devido ao tamanho pequeno e à seqüência ordenada das células;

- permitem que o ATM funcione com altas velocidades de transmissão. O padrão mínimo de transferência é de 155Megabits, com taxas mais altas variando de 622Megabits até 2Gigabits. É interessante comparar com o Ethernet cujo padrão mínimo é de 10Megabits, o Fast Ethernet de 100Megabits, o que ainda está abaixo da velocidade mínima do ATM. Embora o Gigabit Ethernet, atualmente, funcione com velocidades de 1 a 10Gigabits, nem sempre seu desempenho é tão bom quanto o ATM. Isto devido àquelas três características que são exclusivas do ATM.

\section{Conceitos básicos do ATM}

\section{- Célula ATM}

O tamanho das células é pequeno e fixo para facilitar a multiplexação de diferentes fluxos de informação através da rede. Isto permite que uma informação de maior importância possa ser priorizada em relação a uma informação de menor relevância sem a necessidade de remanejamentos complexos das unidades de informação. Unidades de informação de tamanho variável aumentam a complexidade dos comutadores da rede. Quanto maior o tamanho da célula, maior o tempo de empacotamento, causando maior atraso de transferência. Ou seja, a principal vantagem da utilização de células de tamanho fixo está na maior facilidade de tratamento dado pelos equipamentos (na maioria, chaves ATM).

O único argumento contra a utilização de células de tamanho pequeno é o grande overhead que o cabeçalho representa, o que diminui a capacidade efetiva de transmissão na rede. Entretanto tal discrepância tem pouca relevância quando se trata de conexões de alta velocidade.

A célula ATM é composta de 53 bytes, sendo 5 destinados ao cabeçalho (header) e 48 aos dados (payload). Os esquemas abaixo ilustram uma representação esquemática na forma de matriz dos dois tipos de célula ATM (para as interfaces UNI e NNI). As colunas correspondem aos bits e as linhas aos bytes. Os 5 bytes de cabeçalho são compostos pelos campos VPI e VCI PT - CLP - HEC. A única diferença entre os dois tipos de célula é a existência na célula UNI do campo GFC que consome 4 bits do campo VPI em relação à célula. Esse campo serve para definir o tipo de controle de fluxo que deve ser usado na transmissão entre os dois equipamentos se comunicando. Células do tipo NNI trafegam através de conexões ligando duas chaves ATM, já as células do tipo UNI trafegam entre outras conexões, por exemplo entre uma chave ATM e uma chave em Ethernet.

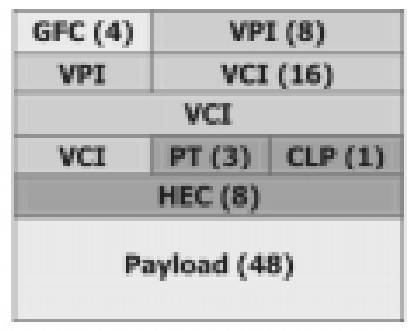

UNI

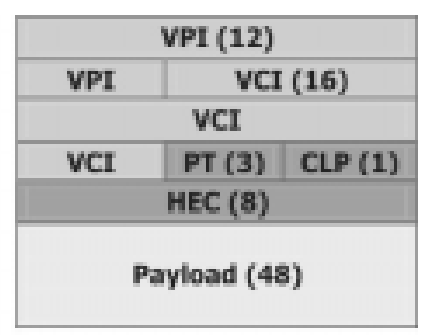

NNI 
- GFC (Generic Flow Control) - definido para uso pelo mecanismo de controle de fluxo genérico.

- VPI (Virtual Path Identifier) - em conjunto com o VCI (Virtual Channel Identifier) formam o rótulo da conexão utilizado pelos comutadores para encaminhar as células ao destino.

- PT (Payload Type) - indica o tipo de informação contida na célula e se a célula passou por elementos congestionados durante seu trajeto.

- CLP (Cell Loss Priority) - indica a prioridade em caso de necessidade de descarte de células.

- HEC (Header Error Check) - usado para detecção de erros no cabeçalho.

- Circuitos Virtuais

O envio de dados em uma rede ATM ocorre sobre circuitos virtuais estabelecidos através da estrutura fisica da rede. A cada Canal Virtual (VC Virtual Channel) é atribuído um VCI (Virtual Channel Identifier) e uma determinada qualidade de serviço (QoS)

Canais virtuais são unidirecionais, portanto quando uma aplicação deseja estabelecer uma comunicação bidirecional com outra aplicação em outro ponto da rede, é necessária a criação de dois canais virtuais, um em cada sentido. Tal situação está ilustrada na Figura 1, em que o equipamento A se comunica com o equipamento $B$ através de dois canais virtuais, um no sentido de $A$ para $B$ e outro no sentido de B para A (Muezerie, 2001).

Uma chave ATM pode, facilmente, criar diversos canais simultaneamente Para facilitar o gerenciamento nas chaves ATM foi criado o conceito de Caminho Virtual (VP - Virtual Path), que agrupa diversos canais virtuais com mesmo ponto de terminação. Cada caminho virtual possui um VPI (Virtual Path Identifier) que é atribuído assim que o caminho virtual é estabelecido. Todos os circuitos virtuais que dividem o mesmo caminho virtual possuem o mesmo VPI. Na Figura 1 são ilustrados dois caminhos virtuais (VP1 e VP2), cada um com três canais virtuais ( $\mathrm{VCl}, \mathrm{VC} 2$ e $\mathrm{VC} 3$ ). Note que os $\mathrm{VCI}$ podem ter os mesmos números identificadores, entretanto eles têm identificadores de caminho (VPI) diferentes. Por exemplo, na Figura 1, estão ilustrados o VCl do VP1 e o VC1 do VP2.

A chave ATM mantém atualizada uma tabela de comutação (ilustrada na tabela da Figura 1), que para todas as conexões de entrada (par VPI/VCI) identifica por qual porto a célula deve sair. Assim para cada célula que chega até a chave é verificada a tabela de comutação, definindo qual caminho a célula deve seguir. Uma chave ATM está ilustrada na Figura 1. Nesta situação, a chave ATM desempenha o papel de mudança de caminho virtual (de VP1 para VP2 e vice-versa) para que o equipamento $\mathrm{C}$ se comunique com o equipamento $\mathrm{B}$, uma vez que os equipamentos estão em caminhos virtuais diferentes.
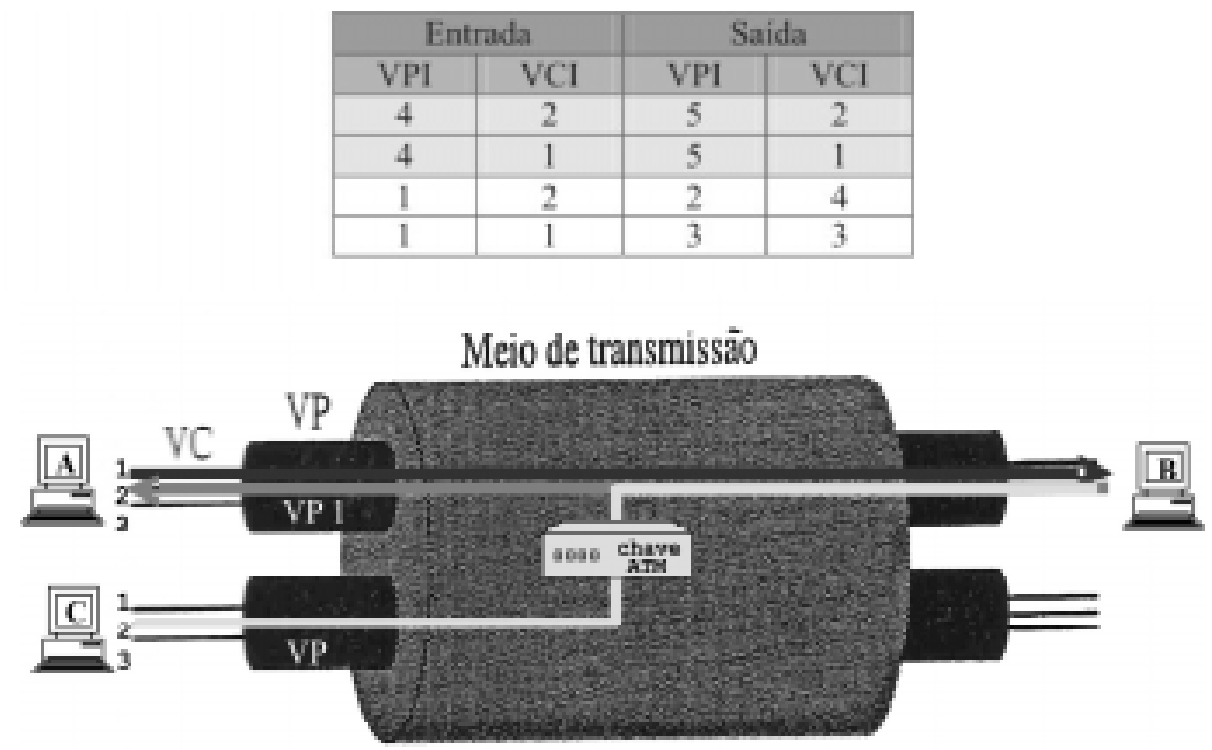

Figura 1. Circuitos e Caminhos Virtuais em ATM (Muezerie, 2001)

- Integrando ATM a outras tecnologias

A tecnologia ATM vem sendo largamente utilizada como backbone de redes locais e de longa distância, também por permitir uma fácil integração com outras tecnologias como Ethernet, Token Ring, Frame Relay, FDDI. O que muitas empresas fizeram foi implantar ATM no backbone sem a necessidade de modificar a rede já instalada, na grande maioria baseada no padrão Ethernet. Estima-se que $80 \%$ das redes mundiais utilizam ATM no backbone (ATM Forum, 2004).

Tal integração é possível devido ao desenvolvimento de diversos protocolos com a finalidade de traduzir certas características intrínsecas a cada tecnologia de forma que elas possam ser incorporadas à tecnologia ATM. Os protocolos mais conhecidos são (Macedo, 1999):

- Classical IP - CLIP: permite roteamento de datagramas IP sobre redes ATM. Como o ATM é orientado à conexão, não existe a emissão de broadcast como acontece em redes baseadas em IP. Para tratar o broadcast das redes IP, o protocolo ATMARP é o responsável.

- LAN Emulation - LANE: emula os serviços das redes Ethernet $\mathrm{e}$ 
Token Ring sobre ATM. Um exemplo é o serviço LES/BUS que emula o ARP e o broadcast do Ethernet.

- Multi-Protocol Over ATM - MPOA: supera as principais desvantagens inerentes aos protocolos CLIP e LANE. Pode ser visto como uma evolução dos protocolos anteriores, acoplando-os em um único protocolo. Provavelmente, será o modo mais comum de integração.

\section{- Qualidade de serviço}

A Qualidade de Serviço $(\mathrm{Q} O S)$ inerente à tecnologia ATM talvez seja a sua mais importante característica. Exatamente o que se espera em transmissões de dados é garantia e qualidade. Para isto, o ATM compreende parâmetros de qualidade de serviço através de recursos de QoS (Quality of Service). Tais parâmetros baseiam-se em certas classes de serviço, em que para cada tipo de transmissão uma determinada classe de serviço pode ser definida. A cada circuito virtual (VC) estabelecido é atribuída uma qualidade de serviço através da definição de uma classe de serviço. Um único computador pode estabelecer vários VCs, cada um com sua respectiva QoS. A Figura 2 ilustra tal situação. Em caso de congestionamento na rede, o VCI 1 pode ser prejudicado enquanto o VCI 2 não será influenciado uma vez que sua largura de banda é garantida pelo QoS definido (no caso, a classe de serviço CBR tem prioridade sobre a UBR). Os tipos de classes de serviço que podem ser definidas são listados abaixo (Macedo, 1999): de voz.

- CBR (Constant Bit Rate): emula uma taxa de bits fixa. Ex: transporte

- rt-VBR (Real Time Variable Bit Rate): a freqüência pode variar porém o máximo atraso e a máxima variação do atraso entre as células é garantido Ex: videoconferência em tempo real.

- nrt-VBR (Non-Real Time Variable Bit Rate): só o valor médio do atraso é especificado. Ex: vídeo gravado

- ABR (Available Bit Rate): a rede tenta maximizar o throughput. Ex: estações de trabalho com interface de rede ATM.

- UBR (Unspecified Bit Rate): não há controle de congestionamento, cada dispositivo pode enviar informação quando desejar e quando houver acúmulo de tráfego. Ex: switch de rede com uplinks ATM.

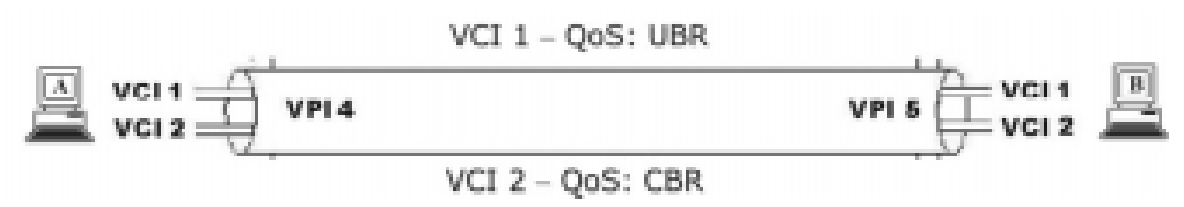

Figura 2. Parâmetros de QoS em canais virtuais

\section{Estudo de casos}

A empresa Marconi (Marconi, 2003), fabricante de equipamentos com a tecnologia ATM, apresenta em seu site uma lista das várias empresas e instituições de ensino que adotaram as soluções da empresa em suas redes ATM. Dentre os vários casos estão a $A T \& T$ Canada, Eurotunnel UK/France, California University of Pennsylvania, Palacky University (Czech Republic), Panama Canal Commision. Dentre as empresas e instituições brasileiras estão a COSIPA, a GlaxoWellcome Brazil e o Laboratório de Sistemas Integrados da Escola Politécnica da USP. Outro caso de utilização bem sucedida da tecnologia está no Projeto Internet2 (Internet2, 2004) no Brasil que se iniciou no ano 2000 .

- Projeto Internet2

A Internet2 é uma versão avançada da Internet que permite transportar áudio, vídeo e dados com garantia de qualidade superior e sem as interrupções comuns da Internet. O projeto se iniciou no Brasil no ano 2000. Ela tornou-se uma realidade no Brasil depois da inauguração da RMAV-SP (Rede Metropolitana de Alta Velocidade de São Paulo), da REMET (Campinas) e da Segunda Fase da Advanced ANSP (a rede acadêmica de São Paulo) realizada em 17 de dezembro de 1999 pelo governador do estado de São Paulo (RMAV-SP, 2004).

A Internet2 (Silveira, 2000) é baseada em tecnologias de alta velocidade, com suporte a aplicações multimídia e requisitos de qualidade de serviço e possui uma infra-estrutura capaz de transmitir dados com velocidade de 155 Mbps. A solução adotada para o projeto recaiu sobre a tecnologia ATM devido às necessidades de alta velocidade com aplicações múltiplas (vídeo, voz e dados) com garantia e qualidade de serviço. $\mathrm{O}$ grande objetivo do projeto é levar às comunidades suporte a aplicações multimídia, como Educação à Distância, Telemedicina e Vídeoconferência.

Atualmente, são 23 universidades e 70 instituições interconectadas. Essas RMAVs nos diversos estados brasileiros constituirão o embrião da Internet2 no Brasil (ver mapa na Figura 3). As primeiras conexões ativas entre São Paulo, Rio de Janeiro, Brasília e Belo Horizonte já estão em operação. A previsão é que esta rede deve ser conectada à Internet2 americana (já em plena operação) através de cabos ópticos partindo do Rio de Janeiro (ver mapa na Figura 3).

Em São Paulo, com enlaces de 155 Mbps estão interconectadas, inicialmente (ver diagrama na Figura 3):

- CCE-USP - Centro de Computação Eletrônica da Universidade de São Paulo.

- INCOR - Instituto do Coração do Hospital das Clínicas.

- UNIFESP - Escola Paulista de Medicina da Universidade Federal de São Paulo. 
- PUC-SP - Departamento de Ciência da Computação da PUC de São Paulo.

- NET - Operadora de TV a Cabo de São Paulo.

- TELEFONICA - Telefônica de São Paulo.

- FAPESP - Fundação de Apoio ao Ensino de São Paulo
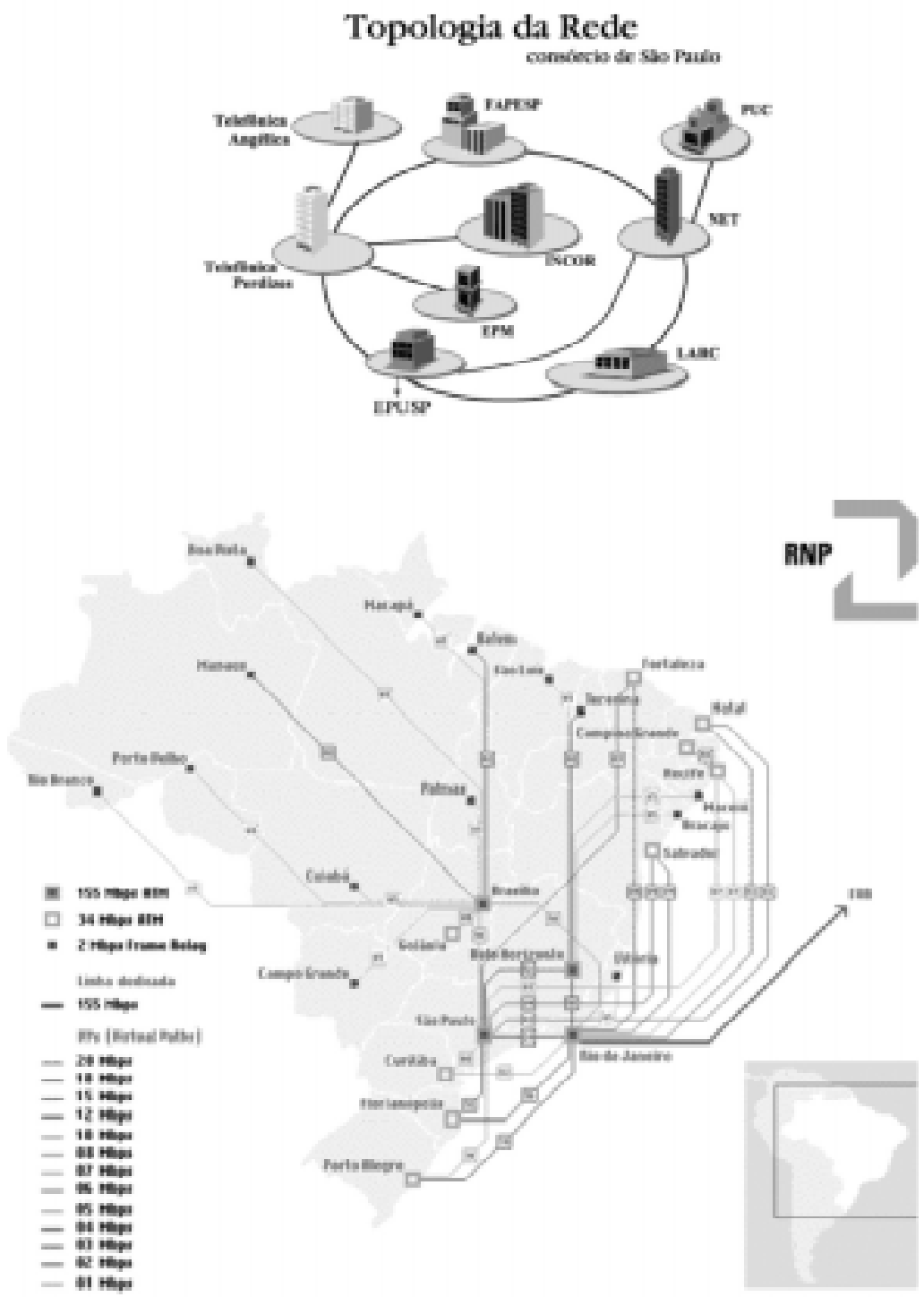

Figura 3. Projeto Internet2 - figuras extraídas de (RMAV-SP, 2004).

\section{Considerações finais}

O mais importante no momento de optar pela adoção de uma tecnologia de redes é conhecer as vantagens e desvantagens de cada uma. O quadro comparativo, mostrado na Tabela 1 , aborda as vantagens e desvantagens para cada uma das duas tecnologias, ressaltando que existem empresas/instituições em que a melhor opção seria adotar a tecnologia ATM, já para outras a tecnologia Gigabit Ethernet seria mais apropriada.

\begin{tabular}{|c|c|c|}
\hline & ATM & Gtgabit Enhernat \\
\hline Complesidade & mais compleso & 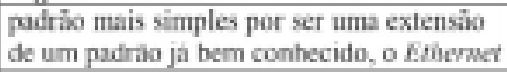 \\
\hline Custo & implamtaçlo mais cara & equipunnentos mais baratos \\
\hline $\begin{array}{l}\text { Suporte a diferentes } \\
\text { tipos de midia }\end{array}$ & melhor intęraçâ0 & $\begin{array}{l}\text { suporta diferemtes tipes de midia mas com } \\
\text { desempenho inferior an ATM }\end{array}$ \\
\hline Estalabilidade & $\begin{array}{l}\text { infinitamente escalivel. tanto em } \\
\text { nûmero de nós na tede quarno em } \\
\text { largura de bands }\end{array}$ & $\begin{array}{l}\text { vem limitaçoes, principalnente no quesino } \\
\text { veloeidade }\end{array}$ \\
\hline Quali & $\begin{array}{l}\text { Q0S nativa garimtia total Bo } \\
\text { transporte }\end{array}$ & $\begin{array}{l}\text { QoS thĩo naiva limitaçues devido à } \\
\text { estrutura da tecrobogia }\end{array}$ \\
\hline dade & $\begin{array}{l}\text { Sticil imegrayajo eom fodas } \\
\text { teenalogias }\end{array}$ & integraçào apenas com o padräo \\
\hline Velocidade & $\begin{array}{l}\text { velocidade nominal de } 155 \mathrm{Mbps} \\
\text { a } 2 \mathrm{Gbps}\end{array}$ & velocidade nonvinal de IGtpss a LOGibps \\
\hline ese & $\begin{array}{l}\text { melhoe desempenho, apesar da } \\
\text { velocidade sominal, as vezes, } \\
\text { inferior }\end{array}$ & $\begin{array}{l}\text { desempenho inferior as ATM, apesar de, } \\
\text { em algums casos, ter maior velocidade } \\
\text { nominal }\end{array}$ \\
\hline
\end{tabular}

Tabela 1. Quadro comparativo ATM x Gigabit Ethernet

\section{Referências bibliográficas:}

ATM FORUM. Disponível em: <http://www.atmforum.com>. Acesso em: 10 abr. 2004.

INTERNET2. Disponível em: $<$ http://www.internet2.edu $>$. Acesso em: 12 maio 2004

MACEDO, C.G.F.; BRAGA, Nilton C.N.C.; ALVES Jr., N. Tutorial: Redes ATM. I Workshop do Rio de Janeiro em Redes de Alta Velocidade, CBPF-NT-005/99, Centro Brasileiro de Pesquisas Físicas, RJ, 1999.

MARCONI. Disponível em: <http://www.marconi.com>. Acesso em: 15 out 2003 
MUEZERIE, A. Caracterização de desempenho de uma rede ATM

Dissertação (Mestrado) - IFSC, USP, São Carlos, 2001. 103 p

RMAV-SP. Disponível em: <http://rmav-sp.larc.usp.br>. Acesso em: 12 maio 2004.

SILVEIRA, R.M. et al. Implantação e utilização da Internet2 de São Paulo. II Workshop RNP2, RJ, 23/24 de maio de 2000. Disponível em:

$<\mathrm{http} / / /$ www.rnp.br/remav/doc_artigos.html $>$.

WEB PROFORUMS. ATM Fundamentals. Disponível em: $<$ http://

www.iec.org/tutorials/atm fund $>$. Acesso em: 10 abr. 2004.

\section{Resumo:}

A tendência atual de integração de serviços de dados, voz e vídeo, estimulada pelo pleno sucesso da Internet, aumentou a demanda por maior banda e melhor desempenho nas redes de comunicação de dados. Neste contexto, a tecnologia ATM (Asynchronous Transfer Mode) vem sendo utilizada na implementação de backbone de LANs e WANs, justamente por oferecer a possibilidade de integração de serviços com qualidade, alta escalabilidade e altas taxas de transferência em banda larga. Neste artigo serão abordados os principais conceitos relacionados à tecnologia ATM, suas vantagens e desvantagens em face de outras tecnologias (como Fast e Gigabit Ethernet), além de casos de sua utilização em empresas e instituições de ensino.

\section{Palavras-chave:}

ATM, Gigabit Ethernet, Backbone, QoS. 